\title{
Carbon ion reirradiation compared to intensity-modulated re-radiotherapy for recurrent head and neck cancer (CARE): a randomized controlled trial
}

Thomas Held ${ }^{1,2,3}$, Kristin Lang ${ }^{1,2,3}$, Sebastian Regnery ${ }^{1,2,3}$, Katharina Weusthof ${ }^{1,2,3}$, Adriane Hommertgen ${ }^{1,2}$, Cornelia Jäkel ${ }^{1,2}$, Eric Tonndorf-Martini ${ }^{1,2}$, Johannes Krisam ${ }^{4}$, Peter Plinkert ${ }^{5}$, Karim Zaoui ${ }^{5}$, Christian Freudlsperger ${ }^{6}$, Julius Moratin ${ }^{6}$, Jürgen Krauss ${ }^{7}$, Semi B. Harrabi ${ }^{1,2,3,8,9,10}$, Klaus Herfarth ${ }^{1,2,3,8,9,10}$, Jürgen Debus ${ }^{1,2,3,8,9,10}$ and Sebastian Adeberg ${ }^{1,2,3,8,9,10^{*}}$ (D)

\begin{abstract}
Background: Intensity-modulated re-radiotherapy (relMRT) has been established as a standard local treatment option in patients with non-resectable, recurrent head and neck cancer (rHNC). However, the clinical outcome is unfavorable and severe toxicities ( $\geq$ grade III) occurred in 30-40\% of patients. The primary aim of the current trial is to investigate carbon ion reirradiation (reCIRT) compared to reIMRT in patients with rHNC regarding safety/toxicity as well as local control, overall survival (OS), and quality of life (QoL).
\end{abstract}

Methods: The present trial will be performed as a single center, two-armed, prospective phase II study. A maximum of 72 patients will be treated with either reIMRT or reCIRT to evaluate severe ( $\geq$ grade III) treatmentrelated toxicities (randomization ratio 1:1). The primary target value is to generate less than $35 \%$ acute/subacute severe toxicity ( $\geq$ grade III), according to the Common Terminology Criteria for Adverse Events v5.0, within 6 months after study treatment. The total dose of reirradiation will range between 51 and 60 Gy or Gy (RBE), depending primarily on the radiotherapy interval and the cumulative dose to organs at risk. Individual dose prescription will be at the discretion of the treating radiation oncologist. The local and distant progression-free survival 12 months after reirradiation, the OS, and the QoL are the secondary endpoints of the trial. Explorative trial objectives are the longitudinal investigation of clinical patient-related parameters, tumor parameters on radiological imaging, and blood-based tumor analytics.

Discussion: Recent retrospective studies suggested that reCIRT could represent a feasible and effective treatment modality for rHNC. This current randomized prospective trial is the first to investigate the toxicity and clinical outcome of reCIRT compared to reIMRT in patients with rHNC.

(Continued on next page)

\footnotetext{
* Correspondence: sebastian.adeberg@med.uni-heidelberg.de

'Department of Radiation Oncology, Heidelberg University Hospital, Im Neuenheimer Feld 400, 69120 Heidelberg, Germany

${ }^{2}$ Heidelberg Institute of Radiation Oncology (HIRO), Heidelberg, Germany

Full list of author information is available at the end of the article
}

(c) The Author(s). 2020, corrected publication 2020. Open Access This article is licensed under a Creative Commons Attribution 4.0 International License, which permits use, sharing, adaptation, distribution and reproduction in any medium or format, as long as you give appropriate credit to the original author(s) and the source, provide a link to the Creative Commons licence, and indicate if changes were made. The images or other third party material in this article are included in the article's Creative Commons licence, unless indicated otherwise in a credit line to the material. If material is not included in the article's Creative Commons licence and your intended use is not permitted by statutory regulation or exceeds the permitted use, you will need to obtain permission directly from the copyright holder. To view a copy of this licence, visit http://creativecommons.org/ licenses/by/4.0/. The Creative Commons Public Domain Dedication waiver (http://creativecommons.org/publicdomain/zero/1. $0 /$ applies to the data made available in this article, unless otherwise stated in a credit line to the data. 
(Continued from previous page)

Trial registration: ClinicalTrials.gov; NCT04185974; December 4th 2019.

Keywords: Head and neck cancer, Reirradiation, Toxicity, Local control, Particle therapy, Heavy ions, Squamous cell carcinoma, Intensity-modulated radiotherapy

\section{Background}

Around $30-50 \%$ of patients with locally advanced head and neck cancer (HNC) develop local recurrence or tumor progression after initial multimodal therapy $[1,2]$. Local tumor growth can lead to severe symptoms such as dysphagia, cachexia, and tumor pain with a significant decrease of the quality of life (QoL). The therapeutic options are limited in this highly pre-treated, vulnerable patient cohort. Depending on the tumor localization, salvage surgery should be evaluated in patients with good performance status with a 2-year progression-free survival around $70 \%$ for stage I/II and 30\% for stage III rHNC [3]. However, only a subset of patients (approximately $30-50 \%$ ) is suitable for a salvage surgery in the clinical routine. Palliative systemic therapies showed limited positive effects with the "EXTREME" regimen resulting in overall survival (OS) rates of 10.1 months and around $80 \%$ severe treatment toxicity ( $\geq$ grade III) [4]. Recently, immunotherapy has emerged as a treatment option for patients with recurrent or metastatic head and neck squamous cell carcinoma (HNSCC) [5].

The clinical routine for reirradiation of $\mathrm{rHNC}$ is reIMRT [6, 7]. Definitive reIMRT is commonly applied with a total dose up to $60 \mathrm{~Gy}$ in 2 Gy fractions [8]. In this heavily pretreated patient cohort, reIMRT was associated with unfavorable rates of treatment-related toxicity ( $\geq$ grade III) in $30-40 \%$ of patients [8-10]. The combination treatment of chemotherapy with photon reirradiation further intensified severe toxicities $[6,7]$ but showed unfavorable 2-year OS rates ranging from 15 to $26 \%$ in patients with rHNC. Stereotactic body radiation therapy (SBRT) has emerged as a viable therapeutic option for non-resectable rHNC with the potential to significantly reduce acute and chronic treatment associated toxicity $[11,12]$. However, the clinical benefit of reirradiation with SBRT compared to reIMRT has yet to be demonstrated in a prospective clinical trial. High total doses from previous irradiation treatment generally limit the dose of reirradiation. The relative biological effectiveness (RBE) of carbon ions is higher compared to photons and it is associated with favorable effects in radioresistant tumors [13]. Because of the steep dose gradient, reCIRT is superior in sparing normal tissue and organs at risk, compared to reirradiation with photons. Consequently, carbon ions possibly represent a feasible and effective treatment modality for salvage reirradiation. A retrospective analysis on 229 consecutive patients with rHNC treated at our clinic with reCIRT showed encouraging results with $3.1 \%$ acute and $14.5 \%$ late severe ( $\geq$ grade III) toxicity [14]. A median total dose of $51 \mathrm{~Gy}(\mathrm{RBE})$ in 17 fractions of $3 \mathrm{~Gy}$ (RBE) was safe and effective for reCIRT with a median OS of 13.7 months for patients with recurrent HNSCC. Data on reCIRT in patients with rHNC is rare, especially in the prospective setting. In the current CARE trial, the impact of reCIRT or reIMRT will be evaluated and the toxicity/safety and efficacy will be compared.

\section{Methods/design}

The trial will be performed as a single-center, twoarmed, randomized controlled phase II study. A maximum of 72 patients are projected to be enrolled into the study. Patients fulfilling the inclusion criteria will be treated with either reIMRT or reCIRT to evaluate severe ( $\geq$ grade III) acute and subacute treatment-related toxicities (randomization ratio 1:1). Randomization will be stratified with respect to histology (HNSCC vs. others) and radiotherapy (RT) interval ( $\geq 2$ years vs. $<2$ years). Block randomization with varying block lengths will be performed. The overall duration of the trial is scheduled to be 60 months, consisting of 48 months of recruitment and a minimum follow-up of 12 months. A flow chart for trial subjects is shown in Fig. 1.

\section{Inclusion criteria}

Inclusion criteria are as follows: Locally recurrent/progressive head and neck cancer after initial radiation therapy; microscopic or macroscopic tumor after salvage surgery; indication for reirradiation; completed wound healing after surgical intervention; Karnofsky Performance Score $\geq 60$; age $\geq 18$ years; written informed consent; ability of subject to understand character and individual consequences of the trial; for women of childbearing potential (and men), adequate contraception; and submission of previous RT records.

\section{Exclusion criteria}

The exclusion criteria are as follows: Reirradiation of malignancy in the larynx; diagnosed plasymocytoma, sarcoma, or chordoma; previous reirradiation in-field; time interval $<6$ months after initial RT; distant metastases (except pulmonary metastases); patients who have not recovered from acute toxicities of previous therapies; refusal of the patients to take part in the study; pregnant 


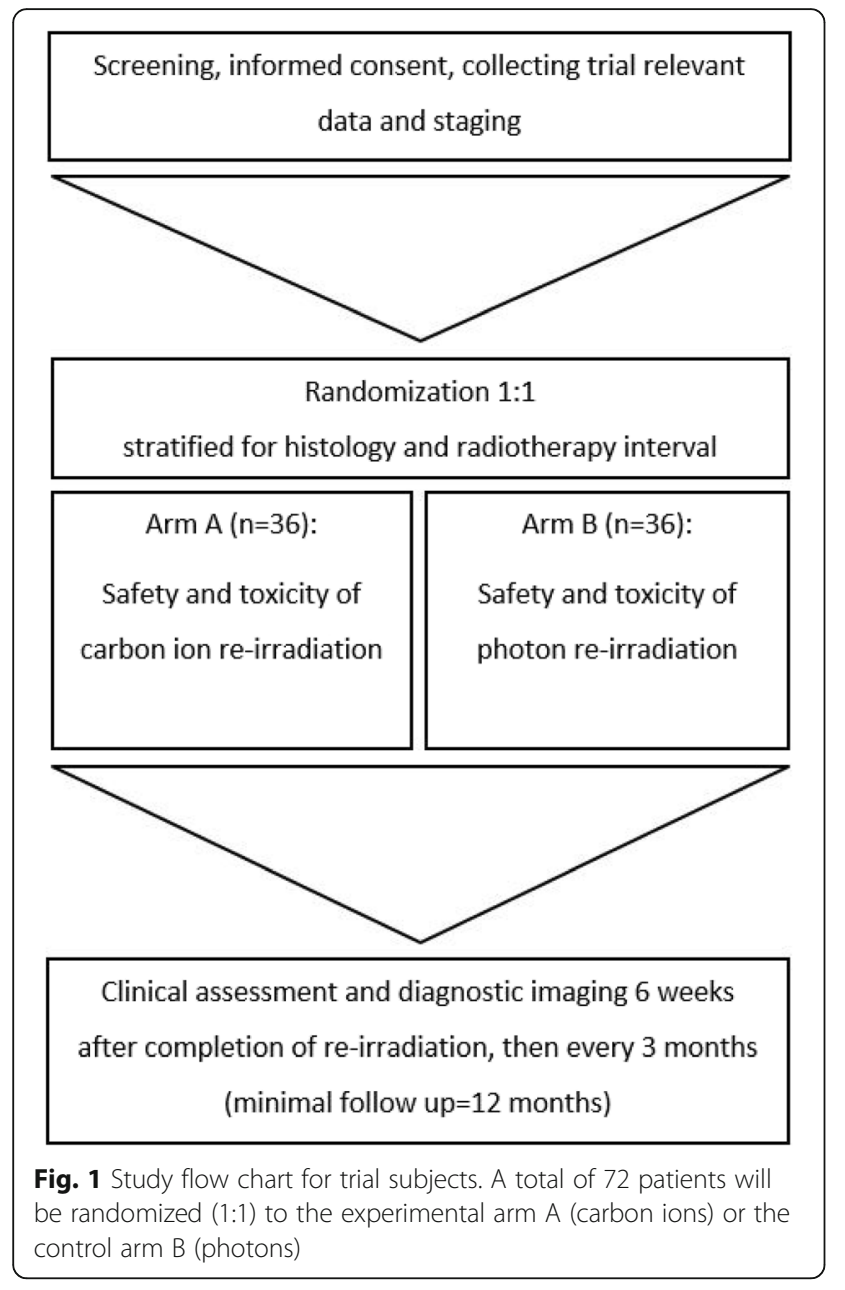

or lactating women; known carcinoma $<5$ years ago (excluding carcinoma in situ of the cervix, basal cell carcinoma, squamous cell carcinoma of the skin) requiring immediate treatment that would interfere with the study therapy; and participation in another clinical study or observation period in a competing trial.

\section{Radiation therapy}

Intensity controlled active raster scanning technology will be used for the application of reCIRT under image guidance with orthogonal $\mathrm{X}$-rays and a daily position correction. A total of 17-20 fractions of 3 Gy (RBE) will be applied on 5-6 days per week, resulting in a total dose of reCIRT ranging between 51 and 60 Gy (RBE). According to standard procedures at our clinic, reIMRT will be performed under image guidance with daily computed tomography $(\mathrm{CT})$ imaging and position correction. A total of 27-30 fractions of 2 Gy will be applied 5 days per week over a period of approximately 5-6 weeks using volume modulated arc therapy (VMAT). Elective nodal irradiation, which is associated with greater risk of acute toxicity, will not be performed.

\section{Treatment planning}

For reirradiation, patients will be immobilized using an individual immobilization mask. All patients will receive a non-contrast planning CT scan in $3 \mathrm{~mm}$ layer thickness and if possible, also contrast-enhanced CT or magnetic resonance imaging (MRI) for optimal target volume definition. Treatment planning will be conducted using the planning software Syngo PT-Planning (Siemens, Erlangen, Germany) including a biologic plan optimization for photon plans and Masterplan Oncentra MasterPlan ${ }^{\circ}$ (Nucletron, Columbia, SC, USA), RayStation $^{\circ}$ (RaySearch Laboratories, Stockholm, Sweden) or Accuray Precision ${ }^{\circ}$ Treatment Planning (Accuray, Sunnyvale, CA, USA) for photon plans. If available, the initial treatment plans will be imported in the planning software. A sum plan of the initial dose distribution and the reirradiation plan will be generated.

\section{Dose prescription}

The total dose of reirradiation will range between 51 and 60 Gy or Gy (RBE) based on clinical experience in reCIRT of $\mathrm{rHNC}$ at our institution within the last 10 years [14]. Patients with an RT interval of less than 2 years will receive $51 \mathrm{~Gy}$ (RBE) reCIRT in $3 \mathrm{~Gy}$ (RBE) or $54 \mathrm{~Gy}$ photons in $2 \mathrm{~Gy}$. Patients with an RT interval of over 2 years can receive up to $60 \mathrm{~Gy}$ (RBE) reCIRT in 3 Gy (RBE) or 60 Gy photons in 2 Gy. Additional factors influencing the dose prescription include the total dose of the initial course of RT, the patient's performance status, initial tumor localization, the tumor volume to be treated with reirradiation, and previous salvage surgery. Individual dose prescription will be at the discretion of the treating radiation oncologist. The maximum total dose of reirradiation for selected patients equals $60 \mathrm{~Gy}$ or Gy (RBE). Details on the dose specifications are summarized in Table 1.

The total dose will be prescribed to the maximum of the calculated dose distribution for the target volume. Treatment planning aims in the coverage of the clinical target volume (CTV) by the 95\%-isodose-line for reCIRT and $90 \%$ coverage of the planning target volume (PTV) for photon plans. The relative biological effective dose of reCIRT will be calculated from the physical dose considering the local effect model LEM1 [13]. BED2Gy refers

Table 1 Dose specification for reIMRT and reCIRT

\begin{tabular}{lll}
\hline & CTV relMRT & CTV reCIRT \\
\hline Dose per fraction & 2 Gy & 3 Gy (RBE) \\
Total dose & $54-60$ Gy & $51-60$ Gy (RBE) \\
BED2Gy $^{a}$ & $/$ & $64-75$ Gy
\end{tabular}

Abbreviations: reIMRT intensity-modulated re-radiotherapy, reCIRT carbon ion reirradiation, $C T V$ clinical target volume, $R B E$ relative biological effectiveness, $B E D 2 G y$ biological effective dose in $2 \mathrm{~Gy}$ fractions, ${ }^{a}$ calculated according to the local effect model (LEM I) with an assumed alpha/beta of 2 
to the equivalent dose in conventional fractionation with 2 Gy fractions. As a precaution, the $\alpha / \beta$ value is uniformly set at $2 \mathrm{~Gy}$, declaring the focus on organs at risk and the prevention of severe treatment-related toxicity. Target volumes of reCIRT will be defined as follows:

Gross tumor volume (GTV) Tumor disease on planning CT scan, contrast enhanced CT scan or T1weighted MRI.

Clinical target volume (CTV) Adding 2-5 mm margin to the GTV including the resection cavity.

Plan target volume (PTV) Adding 2-3 mm margin to the CTV depending on patient positioning and beam angles.

\section{Organs at risk}

Organs at risk such as the brain stem $(\alpha / \beta=2)$, the optic system $(\alpha / \beta=3)$, and the spinal cord $(\alpha / \beta=2)$ will be contoured in accordance with clinical standards at our institution. Fractionation effects of particle therapy will be considered for cumulative dose calculation for organs at risk. Dose constraints for normal tissue and organs at risk in patients with an RT interval of less than 2 years will be respected according to Quantitative Analyses of Normal Tissue Effects in the Clinic (QUANTEC) [15, 16]. Approximate values for symptomatic necrosis of the brain, brainstem, spinal cord myelopathy, and optic neuropathy are listed as less than $3,5,1$, and $3 \%$, respectively, after RT [15]. Data on the tolerance of normal tissue and organs at risk for reirradiation are rare. Preclinical studies on reirradiation of rhesus monkeys suggest substantial recovery of the cervical spinal cord within 2 years after the initial course of RT [17]. At our institution, an increase of the dose tolerance of organs at risk in the second course of irradiation by $20 \%$ after 2 years has shown acceptable safety and treatmentassociated toxicity in retrospective analyses [14]. Dose constraints of organs at risk will be closely guided by the clinical experience at our institution. The corresponding dose volume histograms for the organs at risk should not exceed the proposed values shown in Table 2.

In some cases, sparing of certain organs at risk (e.g. the ipsilateral optic nerve) is not feasible because of direct tumor infiltration. If neurological deficits are expected, because adherence to maximum dose constraints in the respective organs at risk is not reasonably achievable, the patient will be informed in detail. Should the patient accept respective impairments (e.g. vision loss) to achieve local tumor control, written documentation must be obtained before reirradiation. Treatment-related toxicities that occur as a consequence will be evaluated separately.

\section{Study endpoints}

The feasibility/safety of reCIRT in patients with $\mathrm{rHNC}$ is the primary endpoint of the trial. The primary target value is to generate less than $35 \%$ acute/subacute severe toxicity ( $\geq$ grade III) associated with study treatment within 6 months after reirradiation, according to Common Terminology Criteria for Adverse Events (CTCAE) v5.0. The local and distant progression-free survival 12 months after reirradiation, the OS, and the QoL are the secondary endpoints of the trial. Explorative trial objectives are the longitudinal investigation of clinical patientrelated parameters, tumor parameters on radiological imaging, and blood-based tumor analytics.

\section{Study visits and evaluation criteria}

The follow-up corresponds to the clinical routine and is not study-specific, with the exception of the QoL assessments. The first study visit will take place 6 weeks after reirradiation and thereafter every 3 months within the first year after reirradiation. Treatment response and progression will be defined according to the most recent Response Evaluation Criteria in Solid Tumors (RECIST) $1.1[18,19]$. At the time of relapse after reirradiation, routine histological confirmation will be performed if clinically indicated (not study-specific). Detailed information on the study visits and evaluation criteria are shown in Fig. 2.

Table 2 Proposed radiation tolerance of organs at risk for reirradiation

\begin{tabular}{|c|c|c|c|}
\hline & $\begin{array}{l}\text { Maximum cumulative BED2Gy (RT interval }<2 \\
\text { years) }\end{array}$ & $\begin{array}{l}\text { Maximum cumulative BED2Gy (RT interval } \geq 2 \\
\text { years) }\end{array}$ & Comment \\
\hline Brain stem $(\alpha / \beta=2)$ & 60 & $78(\hat{=}+30 \%)$ & $\begin{array}{l}\text { Maximum } \\
\text { (surface) }\end{array}$ \\
\hline $\begin{array}{l}\text { Optic chiasm }(\alpha / \beta= \\
\text { 3) }\end{array}$ & 54 & $64.8(\hat{=}+20 \%)$ & Maximum \\
\hline Optic nerves $(\alpha / \beta=3)$ & 54 & $64.8(\hat{=}+20 \%)$ & Maximum \\
\hline Spinal cord $(\alpha / \beta=2)$ & 45 & $54(\hat{=}+20 \%)$ & Maximum \\
\hline Other organs at risk & ALARA* & & / \\
\hline
\end{tabular}

Abbreviations: BED2Gy biological effective dose in 2 Gy fractions, $R T$ radiotherapy, ALARA as low as reasonably achievable 


\begin{tabular}{|c|c|c|c|c|c|c|c|}
\hline \multirow[b]{3}{*}{ TIMEPOINT } & \multicolumn{7}{|c|}{ STUDY PERIOD } \\
\hline & \multirow{2}{*}{$\begin{array}{c}\text { Enrolment } \\
-t_{1}\end{array}$} & \multirow{2}{*}{$\begin{array}{c}\text { Allocation } \\
0\end{array}$} & \multicolumn{4}{|c|}{ Treatment and follow up } & \multirow{2}{*}{$\begin{array}{c}\text { Close-out } \\
\boldsymbol{t}_{1+12 \text { months }} \\
\text { (ast follow up) }\end{array}$} \\
\hline & & & $\begin{array}{c}\boldsymbol{t}_{l} \\
\left(\boldsymbol{R} T_{\text {start }}\right)\end{array}$ & $\begin{array}{c}\boldsymbol{t}_{2} \\
\left(\boldsymbol{R} T T_{\text {end }}\right)\end{array}$ & $\begin{array}{c}t_{3} \\
\left(I^{s t} \text { follow up 6-8 }\right. \\
\text { weeks affer } R T)\end{array}$ & $\begin{array}{c}\boldsymbol{t}_{4-x} \\
(3-\text { monthly } \\
\text { follow up) }\end{array}$ & \\
\hline \multirow{4}{*}{$\begin{array}{l}\text { ENROLMENT: } \\
\text { Eligibility screen } \\
\text { Informed consent } \\
\text { Allocation }\end{array}$} & & & & & & & \\
\hline & $\mathrm{X}$ & & & & & & \\
\hline & $X$ & & & & & & \\
\hline & & $\mathrm{X}$ & & & & & \\
\hline \multirow{3}{*}{$\begin{array}{l}\text { INTERVENTIONS: } \\
\text { Arm A: reCIRT } \\
\text { Arm B: reIMRT }\end{array}$} & & & & & & & \\
\hline & & & $\longmapsto$ & $\longrightarrow$ & & & \\
\hline & & & $\longmapsto$ & $\longrightarrow$ & & & \\
\hline \multirow{5}{*}{$\begin{array}{l}\text { ASSESSMENTS: } \\
\text { Clinical assessment } \\
\text { Toxicity assessment } \\
\text { QoL questionnaire } \\
\text { CT/MRI head neck }\end{array}$} & & & & & & & \\
\hline & & & $\mathrm{X}$ & $\mathrm{X}$ & $\mathrm{X}$ & $X$ & $X$ \\
\hline & & & $\mathrm{X}$ & $\mathrm{X}$ & $\mathrm{X}$ & $\mathrm{X}$ & $\mathrm{X}$ \\
\hline & & & $\mathrm{X}$ & $\mathrm{X}$ & $\mathrm{X}$ & $\mathrm{X}$ & $\mathrm{X}$ \\
\hline & $X$ & & & & $X$ & $X$ & $X$ \\
\hline
\end{tabular}

Abbreviations: radiotherapy (RT); carbon ion reirradiation (reCIRT); intensity-modulated re-radiotherapy (reIMRT); quality of life (QoL); computed tomography (CT); magnetic resonance imaging (MRI)

Fig. 2 Schedule of enrolment, interventions, and assessments for the prospective randomized controlled CARE trial (SPIRIT figure)

Patients will be followed-up for at least 12 months after reirradiation to document any acute and subacute CTCAE v5.0 toxicity that is related to study treatment. Patients with a partial follow-up are weighted by the proportion of the follow-up time that is completed. Acute toxicities are defined by the occurrence within the first 90 days after the start of reirradiation. Adverse events occurring after the first 90 days until 6 months after study treatment will be documented as subacute toxicities. Any adverse event emerging more than 6 months after reirradiation will be recorded as a late toxicity. Questionnaires used for QoL assessment will be the European Organisation for Research and Treatment of Cancer (EORTC) Quality of Life Questionnaire (QLQ)-C30 [20] and EORTC QLQ-H\&N35 [21].

\section{Data management and statistics}

The data are collected, managed, and processed electronically in the in-house research database. Statistical analysis is based on the International Conference on Harmonization Guidelines "Structure and Content of Clinical Study Reports" and "Statistical Principles for Clinical Trials".

\section{Power calculation}

Assuming a toxicity rate for the carbon ion arm of $\pi_{\mathrm{Tox}}{ }^{\mathrm{CI}}=0.35$ under the null hypothesis, and a toxicity rate of $\pi_{\mathrm{Tox}}{ }^{\mathrm{CI}}=0.15$ under the alternative hypothesis, it will be possible to reject $\mathrm{H}^{0}: \pi_{\mathrm{Tox}}{ }^{\mathrm{CI}} \geq 0.35$ with a probability of $1-\beta=0.803$ with the planned sample size of $n=36$ patients per arm, taking a potential dropout rate of $10 \%$ into account. Furthermore, assuming a toxicity rate of $\pi_{\mathrm{Tox}}{ }^{\mathrm{Ph}}=0.35$ in the photon arm and $\pi_{\mathrm{Tox}}{ }^{\mathrm{CI}}=0.15$ in the carbon ion arm, a difference between treatment arms can only be demonstrated with the planned sample size and a power of $1-\beta=0.8$ using a chi-squared test at the one-sided significance level of $\alpha=0.15$. A comparison at a one-sided significance level of $\alpha=0.05$ would have required a total of $n=146$ patients (including dropouts), which was deemed to be an unfeasibly high number. The power calculation was done using SAS v9.4 (SAS Institute, Cary, NC, USA).

\section{Analysis of the primary endpoint}

The null hypothesis for the primary (safety) endpoint of the trial is defined as $\mathrm{H}^{0}: \pi_{\mathrm{Tox}}{ }^{\mathrm{CI}} \geq 0.35$ (i.e., the rate of patients with an acute/subacute toxicity CTCAE v5.0 $\geq$ grade III in the carbon ion arm is greater than or equal to 0.35), which is tested against its alternative $\mathrm{H}^{1}$ : $\pi_{\mathrm{Tox}}{ }^{\mathrm{CI}}<0.35$ (i.e., the rate of patients with an acute/subacute toxicity CTCAE v5.0 $\geq$ grade III in the carbon ion arm is less than 0.35). The null hypothesis will be tested at a one-sided significance level of $\alpha=0.05$ using an exact binomial test.

Furthermore, the toxicity rate will be estimated alongside a corresponding 90\% exact Clopper-Pearson confidence interval. A (descriptive) comparison of the primary endpoint between the two treatment groups will be performed using a logistic regression model adjusting for histology (HNSCC vs. others) and RT interval ( $\geq 2$ 
years vs. $<2$ years), using a (descriptive) significance level of $\alpha=0.15$ (one-sided) for the odds ratio of the treatment group. The analysis of the primary endpoint will be based on the safety population, which comprises all patients enrolled who received at least one RT fraction.

\section{Analysis of secondary endpoints}

For the secondary endpoint OS, the 1-year survival rates and the median per group will be tabulated together with the respective 95\% confidence intervals, and a KaplanMeier curve will be calculated. A descriptive stratified logrank test adjusting for histology (HNSCC vs. others) and RT interval ( $\geq 2$ years vs. $<2$ years) will be conducted to compare the treatment groups. The analysis of the secondary endpoints local/distant progression-free survival will be performed analogously to the analysis of the primary endpoint OS. Furthermore, exploratory subgroup analyses will be conducted to assess potential predictors of improved efficacy. The analysis of all efficacy endpoints will be primarily based on the ITT population including all randomized patients, while the PP population containing all patients without major protocol violations will be used for sensitivity analyses. Statistical analysis will be performed using SAS v9.4 or higher (SAS Institute, Cary, NC, USA).

\section{Ethics and safety considerations}

The study protocol, patient information sheet, and declaration of informed consent were approved by the Heidelberg University Ethics Committee (S-708/2018). The clinical trial will be performed in accordance with the current version of the Declaration of Helsinki. The recommendations of Good Clinical Practice (GCP) are taken into account regarding the performance, evaluation, and documentation of this study. The regulations concerning medical confidentiality and data protection are fulfilled.

In the clinical study, ionizing radiation is used for the purpose of medical research on humans according to $\$ 23$ StrlSchV (German Radiation Protection Ordinance). The study was approved by the Federal Office for Radiation Protection.

An independent Data and Safety Monitoring Board (DSMB) will monitor recruitment, reported adverse events and data quality at least once a year. Based on its report, the DSMB will make recommendations to the Principal Investigator (PI) regarding the continuation, modification, or termination of the trial. Adverse events will be monitored and documented according to GCP guidelines.

\section{Discussion}

The primary aim of the current randomized controlled trial is to investigate the toxicity profile of reCIRT compared to reIMRT in patients with rHNC. Previous retrospective studies on reCIRT reported encouraging results regarding safety and toxicity [14, 22]. Compared to reIMRT, the inverted dose profile and the sharp dose gradients of heavy ions allow optimal sparing of normal tissue and organs at risk [23, 24]. Therefore, the maximum allowed BED2Gy is 75 Gy for reCIRT and $60 \mathrm{~Gy}$ for reIMRT, limited by the dose constraints of organs at risk. We hypothesize that by using reCIRT, the cumulative rate of severe treatment-associated side effects can be significantly reduced, compared to reIMRT. The physical advantages of carbon ions are yet to be prospectively correlated with clinical toxicity profiles to validate these findings in patients with rHNC. Since many uncertainties regarding correlations of treatment characteristics with toxicity rates in reirradiation of $\mathrm{rHNC}$ remain, the scientific approach by itself influences the results and conclusions. Recently, the actual overlapping retreatment volume was identified as an indicator for acute treatment toxicity, but not the size of the PTV at reirradiation [25]. It is also unclear whether the point maximum or mean cumulative dose are more relevant for causing side effects and how these factors differ for different organs at risk. When treating patients with rHNC with reIMRT, due to a larger low-dose overlap, the definition of the actual retreatment volume itself is to some point arbitrary. To answer these questions, the original treatment plans therefore are required electronically for comparison or should be reconstructed on the retreatment planning $\mathrm{CT}$. Based on these findings, more useful reirradiation categories, depending among others on the treatment volume overlap, can be defined, which would enhance the comparability of clinical trials. By potentially reducing treatment related toxicity, the patients' QoL could be improved, which will be evaluated with standardized questionnaires in the current trial.

In addition, the study aims to analyze the efficacy of reCIRT compared to reIMRT in patients with rHNC. We hypothesize that reCIRT represents an effective alternative to palliative systemic therapies regarding clinical outcome parameters. Because of physiological advantages of reCIRT, enabling further dose escalation compared to reIMRT, improved local disease control and survival for patients with rHNC are conceivable [26]. Effective dose calculation in reCIRT is conducted with assumptions for different tissue types and models, e.g. the local effect model [13]. However, the relative biological effectiveness model prediction performance and correlations with patient treatment plans are subject to substantial variability [27]. Therefore, more effective dose calculations models for carbon ions combined with verification by clinical outcome evaluation with sufficiently large patient cohorts are required. Nonetheless, reCIRT may overcome radioresistance due to the increased RBE, e.g. by eradicating tumor stem cells [28]. Identifying patients that benefit significantly from 
reCIRT represents a major challenge, since a multitude of factors are relevant. A predictive score for reirradiation could possibly guide clinical decision-making, if for this purpose relevant patient and treatment factors are identified from randomized controlled trials. In the current trial, clinical outcome parameters will be correlated with blood-based analytics and tumor parameters on radiological imaging to further explore and validate our findings. At present, this investigation is the first randomized controlled trial prospectively comparing reCIRT to reIMRT in patients with rHNC.

\begin{abstract}
Abbreviations
relMRT: intensity-modulated re-radiotherapy; rHNC: recurrent head and neck cancer; reCIRT: carbon ion reirradiation; OS: Overall survival; QoL: Quality of life; RBE: Relative biological effectiveness; HNC: Head and neck cancer; HNSC C: Head and neck squamous cell carcinoma; SBRT: Stereotactic body radiation therapy; RT: Radiotherapy; CT: Computed tomography; MRI: Magnetic resonance imaging; CTV: Clinical target volume; PTV: Planning target volume; GTV: Gross tumor volume; LEM: Local effect model; BED2Gy: Biological effective dose in 2 Gray fractions; CTCAE: Common Terminology Criteria for Adverse Events; EORTC: European Organisation for Research and Treatment of Cancer; DSMB: Data and Safety Monitoring Board
\end{abstract}

\section{Acknowledgements}

We thank our clinical trial office the support of this study.

\section{Authors' contributions}

T.H., S.H., and S.A. (principal investigator) developed and planned this trial under the supervision of K.H. and J.D. T.H., K.L., P.P., K.Z., C.F., J.M., J.K., S.H., K.H. and S.A. perform patient treatment and clinical assessments. S.H., E.T. and S.A. are responsible for treatment planning, dosimetry, and plan verification. J.K. is the trial statisticians and responsible for statistical planning and statistical analysis. S.A. is responsible for data management. A.H. and C.J. coordinate the study. All authors read and approved the final manuscript.

\section{Funding}

The current study has received major extramural funding within a competitive and peer-reviewed research grant financed by Deutsche Krebshilfe. Open access funding provided by Projekt DEAL.

\section{Availability of data and materials}

Not applicable.

\section{Ethics approval and consent to participate}

The Ethics Committee of the medical faculty of Heidelberg University approved this study on November 5th 2018 and on August 19th 2019 (S708/2018).

\section{Consent for publication}

Not applicable.

\section{Competing interests}

S.A. received grants from Accuray International Sàrl and Merck Serono GmbH outside the submitted work. J.D. received grants from CRI - The Clinical Research Institue GmbH, View Ray Inc., Accuray International Sàrl, Accuray Incorporated, RaySearch Laboratories AB, Vision RT limited, Merck Serono $\mathrm{GmbH}$, Astellas Pharma $\mathrm{GmbH}$, Astra Zeneca $\mathrm{GmbH}$, Solution Akademie $\mathrm{GmbH}$, Ergomed PLC Surrey Research Park, Siemens Healthcare GmbH, Quintiles GmbH, Pharmaceutecal Research Associates $\mathrm{GmbH}$, Boehringer Ingelheim Pharma GmbH Co, PTW-Freiburg Dr. Pychlau GmbH, Nanobiotix A.A. outside the submitted work. The other authors declare no conflict of interest.

\section{Author details}

'Department of Radiation Oncology, Heidelberg University Hospital, Im Neuenheimer Feld 400, 69120 Heidelberg, Germany. ${ }^{2}$ Heidelberg Institute of Radiation Oncology (HIRO), Heidelberg, Germany. ${ }^{3}$ National Center for Tumor
Diseases (NCT), Heidelberg, Germany. ${ }^{4}$ Institute of Medical Biometry and Informatics (IMBI), Heidelberg University Hospital, Heidelberg, Germany. ${ }^{5}$ Department of Otorhinolaryngology, University of Heidelberg, Heidelberg, Germany. ${ }^{6}$ Department of Oral and Maxillofacial Surgery, University Hospital Heidelberg, Heidelberg, Germany. ${ }^{7}$ Department of Medical Oncology, National Center for Tumor Diseases (NCT), Heidelberg University Hospital, Heidelberg, Germany. ${ }^{8}$ Clinical Cooperation Unit Radiation Oncology, German Cancer Research Center (DKFZ), Heidelberg, Germany. ${ }^{9}$ Heidelberg Ion-Beam Therapy Center (HIT), Heidelberg, Germany. ${ }^{10}$ German Cancer Consortium (DKTK), partner site Heidelberg, German Cancer Research Center (DKFZ), Heidelberg, Germany.

Received: 26 June 2020 Accepted: 21 July 2020

Published online: 05 August 2020

References

1. Bernier J, et al. Postoperative irradiation with or without concomitant chemotherapy for locally advanced head and neck cancer. N Engl J Med. 2004;350(19):1945-52.

2. Cooper JS, et al. Postoperative concurrent radiotherapy and chemotherapy for high-risk squamous-cell carcinoma of the head and neck. N Engl J Med. 2004;350(19):1937-44.

3. Goodwin WJ Jr. Salvage surgery for patients with recurrent squamous cell carcinoma of the upper aerodigestive tract: when do the ends justify the means? Laryngoscope. 2000;110(3 Pt 2 Suppl 93):1-18.

4. Vermorken $\mathrm{JB}$, et al. Platinum-based chemotherapy plus cetuximab in head and neck cancer. N Engl J Med. 2008;359(11):1116-27.

5. Burtness $B$, et al. Pembrolizumab alone or with chemotherapy versus cetuximab with chemotherapy for recurrent or metastatic squamous cell carcinoma of the head and neck (KEYNOTE-048): a randomised, open-label, phase 3 study. Lancet. 2019;394(10212):1915-28.

6. Langer CJ, et al. Phase II study of low-dose paclitaxel and cisplatin in combination with split-course concomitant twice-daily reirradiation in recurrent squamous cell carcinoma of the head and neck: results of radiation therapy oncology group protocol 9911. J Clin Oncol. 2007:25(30):4800-5.

7. Spencer SA, et al. Final report of RTOG 9610, a multi-institutional trial of reirradiation and chemotherapy for unresectable recurrent squamous cell carcinoma of the head and neck. Head Neck. 2008;30(3):281-8.

8. Takiar $V$, et al. Reirradiation of head and neck cancers with intensity modulated radiation therapy: outcomes and analyses. Int J Radiat Oncol Biol Phys. 2016;95(4):1117-31.

9. Riaz N, et al. A nomogram to predict loco-regional control after reirradiation for head and neck cancer. Radiother Oncol. 2014;111(3):382-7.

10. Duprez F, et al. Intensity-modulated radiotherapy for recurrent and second primary head and neck cancer in previously irradiated territory. Radiother Oncol. 2009;93(3):563-9.

11. Roh KW, et al. Fractionated stereotactic radiotherapy as reirradiation for locally recurrent head and neck cancer. Int J Radiat Oncol Biol Phys. 2009; 74(5):1348-55

12. Vargo $\mathrm{JA}$, et al. A prospective phase 2 trial of reirradiation with stereotactic body radiation therapy plus cetuximab in patients with previously irradiated recurrent squamous cell carcinoma of the head and neck. Int J Radiat Oncol Biol Phys. 2015;91(3):480-8.

13. Karger $C P$, Peschke $P$. RBE and related modeling in carbon-ion therapy. Phys Med Biol. 2017:63(1):01tr02

14. Held T, et al. Carbon ion Reirradiation for recurrent head and neck Cancer: a single-institutional experience. Int J Radiat Oncol Biol Phys. 2019;105:803-11.

15. Marks LB, et al. Use of normal tissue complication probability models in the clinic. Int J Radiat Oncol Biol Phys. 2010;76(3 Suppl):S10-9.

16. Bentzen SM, et al. Quantitative analyses of Normal tissue effects in the clinic (QUANTEC): an introduction to the scientific issues. Int J Radiat Oncol Biol Phys. 2010;76(3 Suppl):S3-9.

17. Ang KK, et al. The tolerance of primate spinal cord to re-irradiation. Int $J$ Radiat Oncol Biol Phys. 1993;25(3):459-64.

18. Eisenhauer EA, et al. New response evaluation criteria in solid tumours: revised RECIST quideline (version 1.1). Eur J Cancer. 2009;45(2):228-47.

19. Schwartz LH, et al. RECIST 1.1-update and clarification: from the RECIST committee. Eur J Cancer. 2016;62:132-7.

20. Aaronson NK, et al. The European Organization for Research and Treatment of Cancer QLQ-C30: a quality-of-life instrument for use in international clinical trials in oncology. J Natl Cancer Inst. 1993;85(5):365-76. 
21. Bjordal $\mathrm{K}$, et al. Development of a European Organization for Research and Treatment of Cancer (EORTC) questionnaire module to be used in quality of life assessments in head and neck cancer patients. EORTC quality of life study group. Acta Oncol. 1994;33(8):879-85.

22. Jensen $A D$, et al. Re-irradiation of adenoid cystic carcinoma: analysis and evaluation of outcome in 52 consecutive patients treated with rasterscanned carbon ion therapy. Radiother Oncol. 2015;114(2):182-8.

23. Held T, et al. Dose-Limiting Organs at Risk in Carbon Ion Re-Irradiation of Head and Neck Malignancies: An Individual Risk-Benefit Tradeoff. Cancers (Basel). 2019;11(12):2016.

24. Seidensaal K, et al. Re-irradiation with protons or heavy ions with focus on head and neck, skull base and brain malignancies. Br J Radiol. 2020;93(1107): 20190516.

25. Embring A, et al. Overlapping volumes in re-irradiation for head and neck cancer - an important factor for patient selection. Radiat Oncol. 2020;15(1): 147.

26. Gao J, et al. Salvage carbon-ion radiation therapy for Locoregionally recurrent head and neck malignancies. Sci Rep. 2019;9(1):4259.

27. Mein S, et al. Assessment of RBE-weighted dose models for carbon ion therapy towards modernization of clinical practice at HIT: in vitro, in vivo and in patients. Int J Radiat Oncol Biol Phys. 2020. https://doi.org/10.1016/j. ijrobp.2020.05.041.

28. Chiblak S, et al. Carbon irradiation overcomes glioma radioresistance by eradicating stem cells and forming an antiangiogenic and immunopermissive niche. JCI Insight. 2019;4(2):e123837.

\section{Publisher's Note}

Springer Nature remains neutral with regard to jurisdictional claims in published maps and institutional affiliations.

Ready to submit your research? Choose BMC and benefit from:

- fast, convenient online submission

- thorough peer review by experienced researchers in your field

- rapid publication on acceptance

- support for research data, including large and complex data types

- gold Open Access which fosters wider collaboration and increased citations

- maximum visibility for your research: over $100 \mathrm{M}$ website views per year

At BMC, research is always in progress.

Learn more biomedcentral.com/submissions 